\title{
Protocol
}

\section{Time-Lapse Imaging of Membrane Traffic in Living Cells}

\author{
Erik Lee Snapp and Patrick Lajoie
}

Eukaryotic cells are composed of an intricate system of internal membranes that are organized into different compartments-including the endoplasmic reticulum (ER), the nuclear envelope, the Golgi complex (GC), lysosomes, endosomes, caveolae, mitochondria, and peroxisomes-that perform specialized tasks within the cell. The localization and dynamics of intracellular compartments are now being studied in living cells because of the availability of green fluorescent protein (GFP)fusion proteins and recent advances in fluorescent microscope imaging systems. This protocol describes the use of the confocal laser-scanning microscope (CLSM) for time-lapse imaging of one or more fluorescent markers.

\section{MATERIALS}

It is essential that you consult the appropriate Material Safety Data Sheets and your institution's Environmental Health and Safety Office for proper handling of equipment and hazardous materials used in this protocol.

RECIPE: Please see the end of this article for recipes indicated by $<R>$. Additional recipes can be found online at http://cshprotocols.cshlp.org/site/recipes.

Cells expressing the desired GFP-fusion protein(s)

Imaging medium $<\mathrm{R}>$

Equipment

\section{CLSM}

It is assumed that the investigator is familiar with the basic operation of a CLSM. The investigator should understand both the concepts and the operation of pinholes, scan speed, zoom, detector gain, line averaging, laser power, photobleaching, and collection of a time series. Many CLSMs provide the investigator with a number of options for data collection including image size $(512 \times 512,1024 \times 1024$ pixels, etc.), range of data collection (8 bit, 12 bit, or 16 bit), and file formats. It is important to determine the requirements for the image-analysis software in advance. For example, some image-analysis programs cannot process 12-bit images or can only process PGM (portable graymap) image files instead of TIFF (tagged-image file format) files.

A detailed discussion of the instrumentation can be found in Imaging of Membrane Systems and Membrane Traffic in Living Cells (Snapp and Lajoie 2011a).

Temperature control hardware

Adapted from Live Cell Imaging, 2nd edition (ed. Goldman et al.). CSHL Press, Cold Spring Harbor, NY, USA, 2010.

(C) 2011 Cold Spring Harbor Laboratory Press

Cite this article as Cold Spring Harbor Protoc; 2011; doi:10.1101/pdb.prot066555 
1. Set up the CLSM and its associated hardware.

2. Prewarm the imaging stage to $37^{\circ} \mathrm{C}$ or desired temperature, and warm up the microscope lasers for at least $30 \mathrm{~min}$ to avoid power fluctuations during imaging. Image living cells in imaging medium in a chamber as described in the section on Setting Up the Imaging Chamber, in Imaging of Membrane Systems and Membrane Traffic in Living Cells (Snapp and Lajoie 2011a).

3. Identify the cell of interest using the CLSM. Bring it to the desired focus. Scan an image of the whole cell at the desired excitation light intensity, line averaging, zoom, etc. Modify the pinhole and detector gain for maximal fluorescence signal and minimal pixel saturation (pixel intensities that exceed the detector scale, i.e., $>255$ for an 8 -bit image).

It is essential to minimize saturated pixels, as they represent lost information. Saturated pixels only register as the maximum detector value 255 (for an 8-bit image). Thus, information is lost. Detector gain and offset will vary depending on the concentration of the fluorophore, the laser power, and the width and thickness of the fluorescently labeled organelle or region. It is useful to record the detector gain settings to compare behaviors of cells expressing high versus low amounts of fluorescent protein.

4. Empirically determine conditions (i.e., scan speed, zoom, laser power, and microscope objective) that result in minimal photobleaching of the cell during the time course of imaging. Use imaging software to quantitate fluorescence intensity of the whole cell over the time of the experiment.

Quantitating the fluorescence intensity is necessary for determining the extent to which the whole cell undergoes photobleaching during the experiment. Significant continual fluorescence loss from the whole cell during the recovery adds an extra dimension to data interpretation and ultimately should be avoided as much as possible.

5. Use a $40-\mathrm{mW} 488 / 514-\mathrm{nm}$ argon laser at $45 \%-60 \%$ power with $1 \%-5 \%$ transmission for imaging.

The same conditions have been successfully used with a 25-mW 488-nm argon laser. A 543-nm laser is typically used at 15\%-30\% transmission, and a 633-nm laser is typically used at 1\%-5\% transmission. For rapid processes, cells are usually scanned at 0.798-3 sec per $512 \times 512$ frame with either two-line averaging or no-line averaging. To enhance collection speed, the investigator can reduce the imaging field by limiting imaging to a region of interest ( $R O I)$ around the structures of interest. For very rapid processes, the investigator can employ dual direction scanning on newer CLSMs (i.e., the Zeiss 710, Olympus FV100, and Leica SP5) to effectively double the rate of data collection. For slower processes, the intervals at which fluorescence emission is collected need not be rapid (i.e., 3-sec to several-minute intervals).

See Troubleshooting.

6. Because many trafficking processes are relatively rapid, it is necessary to optimize rapid imaging conditions. To image two different fluorescent markers simultaneously using the CLSM, select a chromophore pair with well-separated absorbance and emission spectra, and then image both chromophores simultaneously onto separate detectors (i.e., a photomultiplier tube).

More recent CLSMs (i.e., the Zeiss 710 and the Leica SP5) provide the investigator with substantial control over fluorescence emission detection and separation. If the investigator is unable to avoid signal bleed through, then image each fluorophore separately. To approximate simultaneous imaging, the newer CLSMs permit line imaging, which alternates laser excitation for each horizontal line of pixels. Alternating whole-image frames will decrease the likelihood of colocalizing two different chromophores on dynamic structures.

7. Collect 10 initial images to establish the baseline fluorescence intensity and distribution. These images can be used to confirm that the fluorescence intensities do not fluctuate significantly.

8. Collect multiple data sets for each experiment to confirm reproducibility and for statistical analyses.

One way to increase data collection, with no loss in resolution, is to configure the CLSM for a $2048 \times 2048$ image collection. At zoom 1, the resulting $2048 \times 2048$ image is equivalent to a zoom-4 $512 \times 512$ image but over an area four times the size of a normal zoom-4 image. The image-collection time will increase. However, the increased area means that the investigator can simultaneously image more cells. 
Problem: There is autofluorescence noise.

Solution: Lysosomes are notorious for autofluorescence when excited with light between 400 and 488 $\mathrm{nm}$. To decrease autofluorescence, lower the intensity of the excitation beam, and use narrower bandpass emission filters. In addition, avoid phenol red and high serum concentrations $(>20 \%)$ in the cell growth medium prior to imaging.

Problem (Step 5): The fluorophore photobleaches too rapidly during image acquisition. Solution: Consider the following:

1. Decrease the excitation light intensity using either neutral-density filters or by lowering the voltage to the acousto-optical modulator, and increase the gain on the detector side to collect light more efficiently.

2. Alternatively, collect images less frequently to reduce laser exposure.

Problem (Step 5): Cells are excited with a constant light intensity, but the fluorescence intensity varies over time.

Solution: If this is not due to a biologically relevant process such as recruitment or degradation of fluorophore, then either the focus is shifting during acquisition or the laser-power output is unstable. Consider the following:

1. Maintain the focus either manually or by using autofocus software, which is available for many CLSMs.

2. If laser output is a concern, then check it by exciting fluorescent beads and quantifying the emission over time. Fluctuating laser output may be due to the laser being operated at too low of a power output. Increasing the power output to 50\% may help. If this fails to correct the problem, then contact your microscope service representative.

Problem (Step 5): Focus drifts.

Solution: Consider the following:

1. Try using relatively flat cells (i.e., COS-7 or U-2 OS), which may alleviate the problem.

2. Try imaging with the pinhole partly or entirely open and use lower NA objectives.

3. Pre-equilibrate the sample to the temperature of the objective, as this will prevent hysteresis (expansion or contraction) of the coverslip or chamber during imaging.

4. Ensure that the stage insert is mounted securely and that the sample is tightly seated in the holder.

Protocols are also available for Photobleaching Regions of Living Cells to Monitor Membrane Traffic (Snapp and Lajoie 2011b) and Activating Photoactivatable Proteins with Laser Light to Visualize Membrane Systems and Membrane Traffic in Living Cells (Snapp and Lajoie 2011c).

Imaging Medium

Phenol-red-free cell growth medium (e.g., RPMI or DMEM)

Fetal bovine serum (10\%)

Glutamine (2 mM)

HEPES (25 mM, pH 7.4) 
Time-Lapse Imaging of Membrane Traffic

\section{REFERENCES}

Snapp EL, Lajoie P. 2011a. Imaging of membrane systems and membrane traffic in living cells. Cold Spring Harb Protoc doi: 10.1101/pdb. top066548.

Snapp EL, Lajoie P. 2011b. Photobleaching regions of living cells to monitor membrane traffic. Cold Spring Harb Protoc doi: 10.1101/pdb. prot066563.
Snapp EL, Lajoie P. 2011c. Activating photoactivatable proteins with laser light to visualize membrane systems and membrane traffic in living cells. Cold Spring Harb Protoc doi: 10.1101/pdb.prot066571. 


\section{Time-Lapse Imaging of Membrane Traffic in Living Cells}

Erik Lee Snapp and Patrick Lajoie

Cold Spring Harb Protoc; doi: 10.1101/pdb.prot066555

\begin{tabular}{|c|c|}
\hline $\begin{array}{r}\text { Email Alerting } \\
\text { Service }\end{array}$ & Receive free email alerts when new articles cite this article - click here. \\
\hline $\begin{array}{r}\text { Subject } \\
\text { Categories }\end{array}$ & $\begin{array}{l}\text { Browse articles on similar topics from Cold Spring Harbor Protocols. } \\
\text { Cell Biology, general (1382 articles) } \\
\text { Cell Culture ( } 301 \text { articles) } \\
\text { Cell Imaging (525 articles) } \\
\text { Confocal Microscopy ( } 114 \text { articles) } \\
\text { Fluorescent Proteins ( } 259 \text { articles) } \\
\text { Image Analysis ( } 124 \text { articles) } \\
\text { Video Imaging / Time Lapse Imaging (171 articles) }\end{array}$ \\
\hline
\end{tabular}

